\title{
Image Compression in Wireless sensor networks- A survey
}

\author{
M.Sheik Dawood \\ Associate Professor \\ Sethu institute of technology \\ Pulloor, Kariapatti, India
}

\author{
L.Ahila \\ PGScholar \\ Sethu institute of technology \\ Pulloor, Kariapatti, India
}

\author{
S.Sadasivam \\ Associate Professor \\ Sethu institute of technology \\ Pulloor, Kariapatti, India
}

\author{
G.Athisha \\ Professor \\ PSNA College of Engg\&tech \\ Dindigul, India
}

\begin{abstract}
Wireless Sensor networks are battery powered due to which their lifetime is precisely limited. In this type of network the nodes commonly have very limited resources in terms of processing power, bandwidth and energy. Efficient coding of the multimedia content is therefore important. One possible way of achieve maximum utilization of those resource is applying compression on sensor event Usually, processing data consumes much less power than transmitting data in wireless medium, so it is effective to apply compression before transmitting data for reducing total power consumption by a sensor node. In this paper various energy efficient image compression techniques Collaborative image transmission using Sobal edge-detection, JPEG2000 image compression, Image Subtraction with Quantization of image; Adaptive Compression and Spatial Correlation-Based Image Compression are discussed.
\end{abstract}

Keywords: Image compression, energy efficiency, sensor network

\section{INTRODUCTION}

Advances in wireless communication have enabled the development of low-cost, low-power visual multihop wireless networks, which have recently emerged for a variety of applications, including environmental and habitat monitoring, target tracking, surveillance and emergency response [1]. A Wireless Sensor Network is a self-configuring network of small sensor nodes communicating among themselves using radio signals, and deployed in quantity to sense, monitor and understand the physical world. Wireless Sensor nodes are called motes. WSN provide a bridge between the real physical and virtual worlds. Allow the ability to observe the previously unobservable at a fine resolution over large spatiotemporal scales. It has a wide range of potential applications to industry, science, transportation, civil infrastructure, and security.

The basic wireless sensor network configuration [2] is that a large number of sensor nodes are densely deployed over a sensor field. All nodes are connected by radio frequency, infrared, or other medium without any wire connection. The data collected by nodes traverses among the nodes in wireless medium. In order to realize WSNs, peer-to-peer network techniques are widely used so that it allows direct communication between any two nodes. If two devices cannot communicate directly, other nodes, located between those two nodes, will transmit a data packet from the source node to the destination node. This is called multi-hop routing. Because of their peer-to-peer communication style, no centralized point, which will control a network formation like a base station for a cellular system, is required for the network. Since no fixed infrastructure is necessary for WSNs, a network will be constructed inexpensively.

Considering compression and transmission of images in a wireless sensor network, then the focus on the design and performance evaluation of distributed image compression algorithms are analyzed. The benefit of using distributed image compression in sensor networks can be illustrated in the following two cases. In the first case, nodes have extremely constrained computation power. Hence, a node does not have sufficient computation power to completely compress a large raw image. In this case, a distributed method to share the processing task is required to overcome the computation power limitation of each single node. In the second case, even if nodes are not extremely computation powers constrained, but are battery operated; distributing the computation load of processing every raw image among otherwise idle processors of other nodes extends the overall lifetime of the network.

Several energy efficient protocols of image compression are proposed for wireless applications. However representing multimedia data requires a large amount of information, leading to high bandwidth, computation energy, and communication energy requirements for mobile multimedia communication. The characteristic of wireless multimedia communication which can be used to overcome the bandwidth and energy may vary. One possible way to achieve maximum utilization of resources is applying data compression to sensor data. In this paper some of image compression schemes specifically designed for WSNs is presented and discussed.

\section{ENERGY CONSUMPTION ANALYSIS IN WIRELESS MEDIUM}

Energy consumption is one of the most important performance metrics for wireless sensor networks because it directly relates to the operational lifetime of the network. Most of the researches are focusing mainly on performance comparisons and trade-off studies between various lowenergy routing and self-organization protocols, while keeping other system parameters fixed. As a result, very little has been revealed about the relationship between the aggregate energy consumption and non-protocol parameters such as node density, network coverage area, and sensor traffic generation and distribution, and transceiver power characteristics.

In terms of power consumption, operation of a wireless sensor node can be divided into three parts: sensing, processing, and transmission. Among those three operations, it is known that the most power consuming task is data transmission. Thus, if we can minimize the size of data by compression, it will reduce transmission power. However, on the other hand by applying data compression more power for processing will be required to perform a compression algorithm. In order to 
reduce total power consumption, the sum of power consumed by transmission and processing has to be reduced. The power consumed by compressing original data string into reduced data string has to be smaller than the power consumed by transmitting difference in bits of data string. Applying the image compression before transmitting in wireless medium is effective to reduce the amount of energy consumption. However, it is crucial to select an image compression algorithm, which requires less memory access during execution time.

\section{IMAGE \\ TECHNIQUES}

Energy optimization in sensor networks is very complicated because it not only involves the reduction of energy consumption but also load distribution in the network. Minimizing data size before transmitting in wireless medium is effective to reduce total power consumption. Energy efficient image communication is one of the most important goals for WSN.

\subsection{Collaborative image transmission using Sobal edge-detection}

A novel distributed image compression is examined in [3] for wireless sensor networks to avoid the extra energy usage during redundant data transmission. First a shape matching method to coarsely register image is applied by sharing shapecontext to avoid communication overhead. Image is divided into sub regions based on the gray scale value and quantization is performed on sub regions. This load is shared on the overlapping camera node. System model for this compression in WSN shown in Fig. 1 and densely deployed sensor network is considered. Image in the neighbor sensor are spatially correlated with overlaps. A common region of overlapped area is identified by sharing low bandwidth descriptors as feature points [6]. This is done in order to reduce the redundant data in the images and hence life time is increased.
The joint compression is implemented using six steps: Find the feature points and broadcast to neighbor nodes; Identifying the camera with overlapping field of view; Find the common area in overlapping camera; Distribute the image to each camera based on gray scale regions; Quantized these regions up to sixteen or less gray level; Reconstruct the final image at destination from small quantized images.

Sobal edge-detection is used to extract the feature points, so that the communication overhead between sensor nodes for can be reduced. Shape contexts have been assigned to the reference image and overlapping image. The next step is to determine the best between reference image and overlapping image. Then the overlapped area is divided into small regions. This division depends upon the gray level, in other words image is divided tilling based on the gray scale value that are within the threshold value. Then each sub region is quantized up to sixteen gray scales and then compressed locally. This local region is sent towards the destination with its quantization value and its coordinate's information. The quantization value is that value on which this small region is quantized at sixteen gray scale values and by using this value the region is reconstructed again.

The discussed approach is feasible for the image that has large regions and low variation. This is due to the quantization based on the regions. It is more feasible for low contrast images.

\subsection{JPEG2000 image compression}

Distributed JPEG2000 image compression scheme is discussed in [4]. In this research the performance of distributed image compression in energy constrained multihop ad hoc and sensor network is explored. By exploiting the characteristics of the Discrete Wavelet Transform (DWT, a distributed JPEG2000 image compression scheme where nodes compress an image while forwarding it to the destination subject to a specific image quality constraint is proposed.
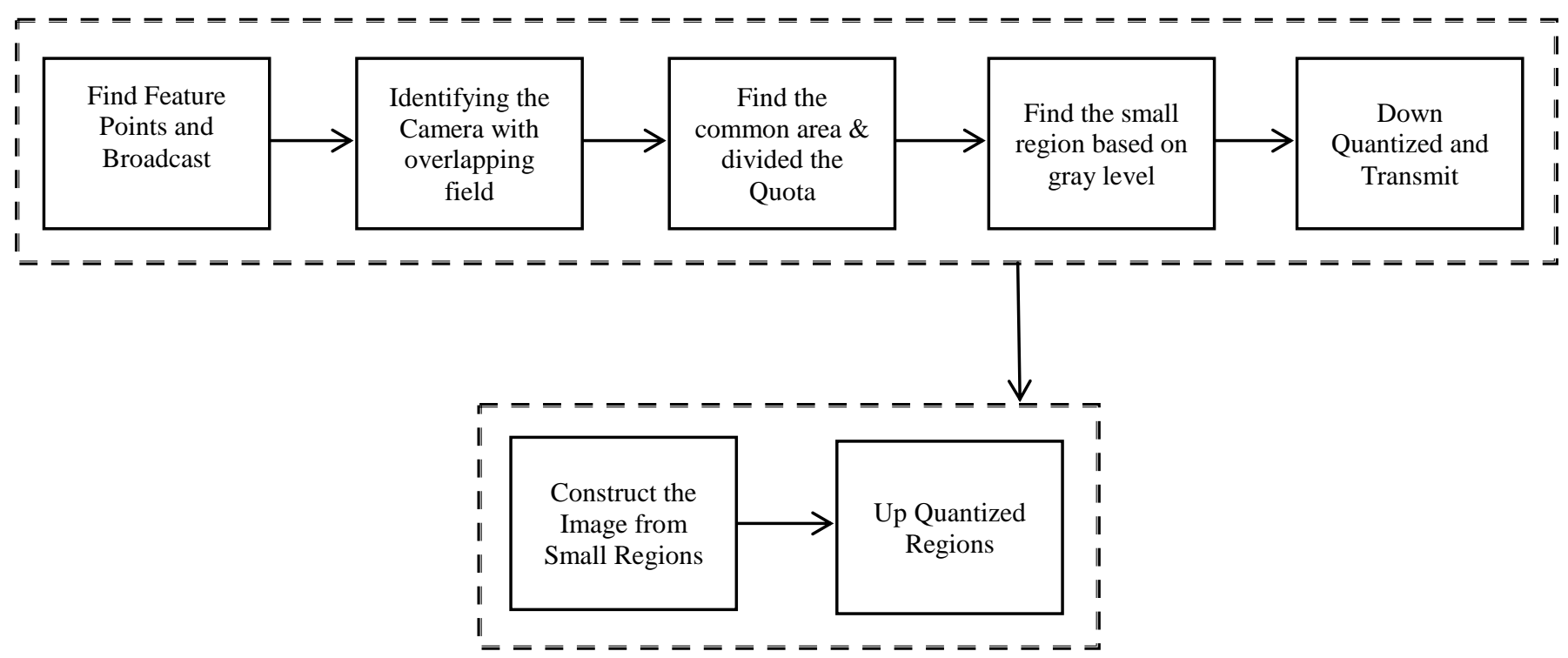

Fig.1: System model 
The basic idea of distributed JPEG2000 image compression is distributing the workload of wavelet transform to several groups of nodes along the path from the source to the destination.

Two approaches of data exchange will be investigated and compared with respect to image quality and energy consumption; 1)Method 1: Divide by rows/columns; 2)Method 2: Tiling. In the first method the data partitioning scheme proposed in traditional parallel wavelet transform is considered when applied to a multihop wireless network. Here data are divided into rows and then one dimensional DWT is performed. Further intermediate results are created when send to node and divided into several blocks. Once again one dimensional DWT is performed and get collected in the nodes.

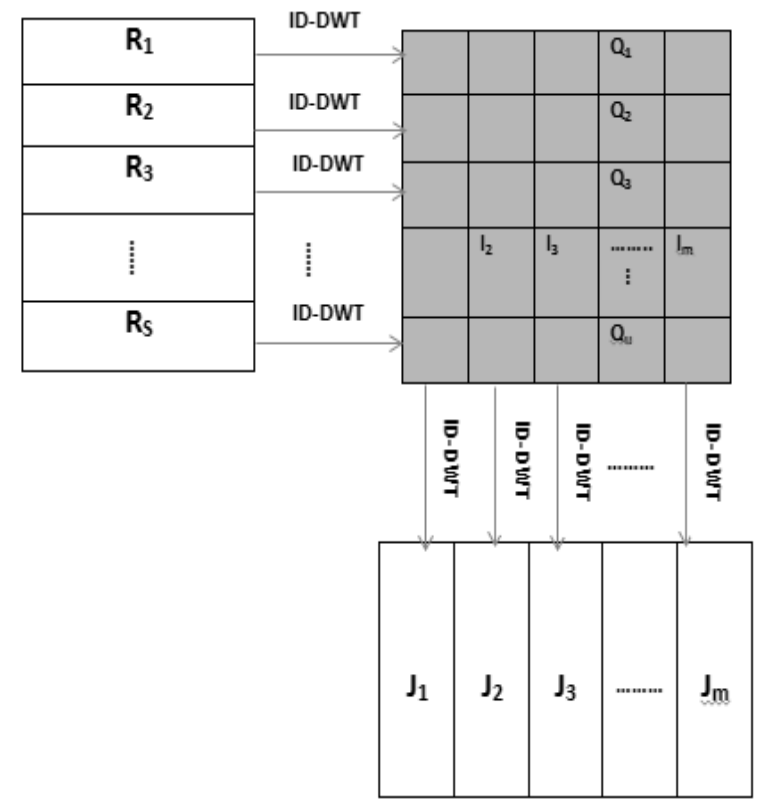

Fig. 2: Traditional data partitioning approach

In the next method Tiling, which is used in JPEG, can also be used in wavelet based image compression. Here data are separated into several blocks and send to node individually for further process. There two dimensional transform is performed. In this compression they have taken small tiles hence distortion loss and blocking artifacts have been decreased.

Then the bit allocation, quantization step and entropy coding method in the proposed distributed image compression use the same method as in the centralized JPEG2000 image compression. Finally load balancing is done.

\subsection{Image Subtraction with Quantization of image}

A novel technique, Image Subtraction with Quantization of image (ISQ) is presented in [5]. In this technique only the changes in the image are sent back instead of sending back the whole image. Then the small changes are extracted from the original images and then send back to the destination by quantizing the small changes. In the destination the images are recomputed and then original reconstructed image is got back.
This scheme is very simple and easy to implement while still satisfying image quality requirement. Simulation results show that our scheme increases the system lifetime by up to ten times. The system structure is shown in Fig.3.

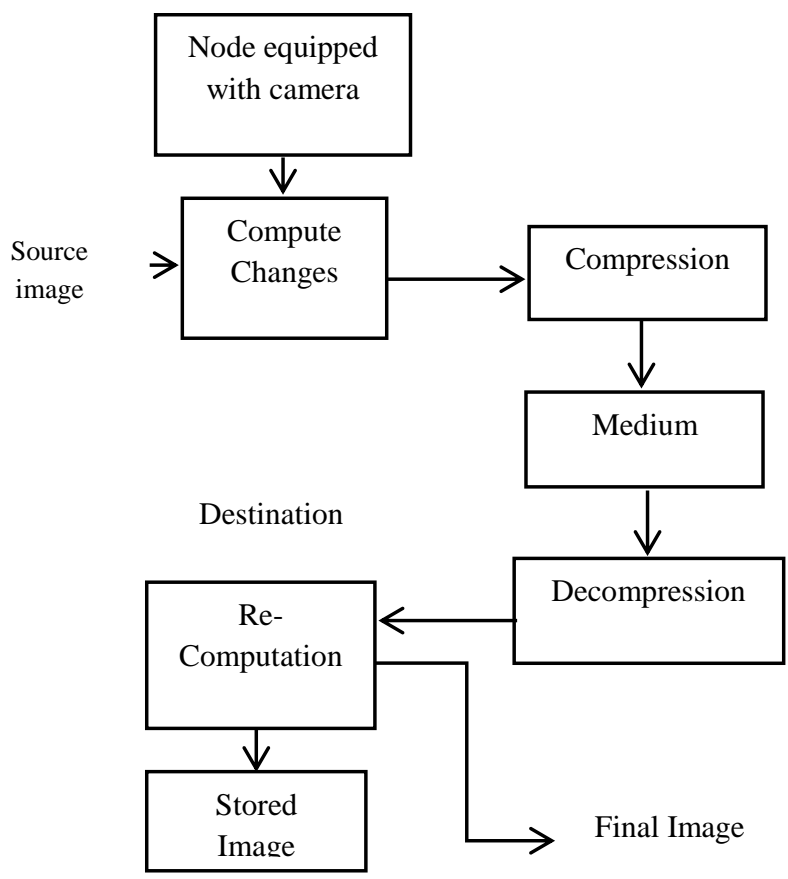

Fig.3: System structure

The system structure shown is divided into three phases, source image side, where an image is generated after query from the destination, and compressed by using the proposed method (ISQ), medium where the compressed image node by node moved towards destination node, and destination side, where decompression is applied according to the proposed method.

As the node is fixed, thus there is no change in new image expect the new body is introduced. So instead of sending the whole image, only changes are sent back. Through simulation it is shown that ISQ improves the energy efficiency of camera equipped node of the sensor networks. The proposed technique is very simple, and easy to implement and gives the better results with respect to processing power.

\subsection{Adaptive Compression}

Adaptive Compression of Slowly Varying Images is discussed in [7]. The technique introduced can be used in applications in which it is essential to retain the transmission of sequential image frames over a WMSN communication link. The proposed scheme is an adaptive image compression algorithm that regulates the quality of the transmitted images according to: (a) the traffic load within the network, and (b) the level of data contained in an image frame. The proposed image transmission scheme is based on the application of the QTD [8] with a varying decomposition compression factor within a gradient adaptive approach. The system architecture of this compression is shown in Fig.4.

QTD is an image segmentation method generally used for hierarchical decomposition. The main idea of hierarchical decomposition is to divide an image into sufficiently homogeneous areas, the levels of which can be compactly 
encoded. QTD has been widely utilized not only due to its low-complexity but because it is as well a powerful compression method. The tree-like evaluation of an image enables the removal of the unnecessary leaves and branches out of the tree, which result in the reduction of the QTD representation size.

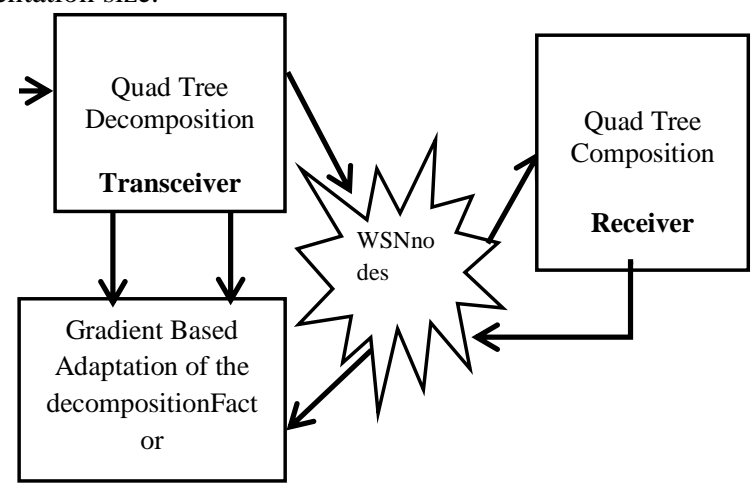

Fig.4: System architecture

First the images captured through a camera are QTdecomposed. Next, the resulting QTD partitioning of each single image is transformed in data packets, in order to enable the image transmission over the employed WMSN. Through the multihop property of WSMNs the data packets reach the receiver side where the received data streams are composed into images.

It should be noted that the proposed scheme addresses only the cases where there is a small variation of the captured image content, such as applications of surveillance and security. This limitation is mandatory for allowing the convergence of the proposed adaptation scheme. In the cases of fast content variations, even if a larger adaptation factor has been adopted, the proposed algorithm will not converge, the obtaining QTD-images will have a very poor quality, and the a priori soft time bounds will not be possible to be met.

\subsection{Spatial Correlation-Based Image Compression}

Spatial correlation-based image compression framework for wireless multimedia sensor networks is discussed in [9]. This framework is proposed mainly to maximize the compression of visual information gathered in a WMSN. This framework consists of two components: 1) compression efficiency prediction and 2) coding hierarchy construction. The compression efficiency prediction aims to estimate the compression gain from joint encoding of multiple cameras before the actual images are captured. To achieve this, an entropy-based divergence measure (EDM) scheme is proposed, which only takes the camera settings as inputs without requiring the statistics of real images.

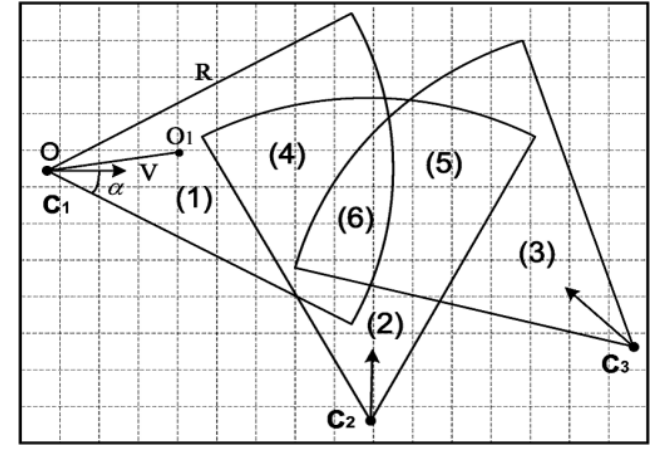

Fig. 5: Field of views of multiple cameras.

In the EDM, the overlapping pattern of the field of views (FoVs) of multiple cameras is first identified. Then, the correlation degree among the observations from cameras with overlapped FoVs is obtained through a spatial correlation model. Based on the correlation characteristics, a dependency graphbased algorithm is designed to estimate thejoint entropy of multiple cameras. This joint entropy effectively predicts the compression performance for joint encoding of multiple cameras. Using the results from EDM, the next problem is how to establish a compression-oriented coding hierarchy, which can achieve a substantial compression gain and decoding reliability.

\section{CONCLUSION}

In this paper various image compression schemes Collaborative image transmission using Sobal edge-detection, JPEG2000 image compression, Image Subtraction withQuantization of image, Adaptive Compression and Spatial Correlation-Based Image Compression are analyzed based on their compliance to wireless sensor network. Compression ratio and power saving manners in these schemes are fairly impressive. They are one of possible method to diminish resource constrain of wireless sensor nodes in combination with energy efficient clustering mechanism.

\section{REFERENCES}

[1] D. Culler, D. Estrin, and M. Srivastava.2004. "Guest Editors' Introduction: Overview of Sensor Network," Computer, Volume: 37 Issue: 8, pp. 41-49.

[2] I.F. Akyildiz, W. Su, Y. Sankarasubramaniam, and E. Cayirci.2002. "A survey on sensor networks," IEEE Communications Magazine, Vol 40 Issue: 8.

[3] M.I.Razzak, S. A. Hussain, Abid Ali Minhas and Muhammad Sher.2010. "Collaborative Image Compression in Wireless Sensor Networks," in Int Journal of Computational Cognition, Vol. 8, No. 1.

[4] Huaming $\mathrm{Wu}$ and Alhussein A. Abouzeid, "Energy Efficient Distributed JPEG2000 Image Compression in Multihop Wireless Networks,"Applications and Services in Wireless Networks, 2004.

[5] S.A.Hussain, M.I. Razzak, A. A. Minhas, M. Sher and G.R Tahir.2009. "Energy Efficient Image Compression in Wireless Sensor Networks," in International Journal of Recent Trends in Engineering, Vol. 2, No. 1. 
[6] R. Wagner, R. Nowak, and R. Baraniuk.2003, "Distributed image compression for sensor networks using correspondence analysis and super-resolution," in Proceedings of IEEE ICIP'03, volume 1, pages 597-600, Barcelona, Spain.

[7] George Nikolakopoulos, DionisisKandris, Anthony Tzes.2010. "Adaptive Compression of Slowly Varying Images Transmitted over Wireless Sensor Networks," Sensors journal, Vol 10,pages 7170-7191
[8] Yi-Chen Tsai, Ming-Sui Lee, MeiyinShen and C.-C. Jay Kuo, .2006. "A quad-tree decomposition approach to cartoon image compression," IEEE International Workshop on Multimedia Signal Processing (MMSP).

[9] Pu Wang, Rui Dai, Ian F. Akyildiz.2011. “A Spatial Correlation-Based Image Compression Framework forWireless Multimedia Sensor Networks," IEEE Transactions on Multimedia, Vol. 13, NO. 2 